\title{
Publisher Correction: Shaping human telomeres: from shelterin and CST complexes to telomeric chromatin organization
}

\author{
Ci Ji Lim (D) and Thomas R. Cech (D)
}

Correction to: Nature Reviews Molecular Cell Biology https://doi.org/10.1038/s41580-021-00328-y, published online 09 February 2021.

In Figure 2b, the label 'TRFH-binding motif' has been nudged down, towards the illustration of TIN2, and re-styled to match the other protein-domain labels in the figure. The changes have been made in the HTML and PDF versions of the article.

https://doi.org/10.1038/s41580-021-00353-x I Published online 19 February 2021

(c) Springer Nature Limited 2021 